\title{
Millimeter-Wave Propagation in Vegetation: Experiments and Theory
}

FELIX K. SCHWERING, SENIOR MEMBER, IEEE, EDMOND J. VIOLETTE, AND RICHARD H. ESPELAND

\begin{abstract}
Microwave/millimeter-wave propagation in woods and forests was investigated at $9.6,28.8$, and $57.6 \mathrm{GHz}$. In order to perform the measurements under well defined, reproducible conditions, a regularly planted, well groomed stand of trees of about the same growth (pecan orchard near Wichita Falls, TX) was chosen as the test site. The experiments were repeated, over the same transmission paths, under both summer and winter conditions, i.e., with trees in leaf and without leaves. Of particular interest were the range dependence, beam broadening, and depolarization of millimeter-wave beams in vegetation and the frequency dependence of these effects. The experiments have shown, in particular, that the range dependence is characterized by a high attenuation rate at short vegetation depths and a reduced attenuation rate at large depth. For trees fully in leaf, the transition between the two regimes can be rather abrupt and the change in attenuation rate substantial. Just after the transition significant beam broadening (and depolarization) occurs.

A theory of millimeter-wave propagation in vegetation was derived using transport theory. Theoretical and experimental results are in good qualitative agreement; both show the same trends. The theory explains these trends in terms of the interplay between the coherent (direct path) field component and the incoherent (multiscattered) field component. Achieving good quantitative agreement will require further refinement of the theory.
\end{abstract}

\section{INTRODUCTION}

$\mathrm{I}$ PRINCIPLE, millimeter-wave links for point-topoint transmission require unobstructed line-of-sight paths. In practical situations, the line-of-sight may run through vegetation over part of its path, in particular if the two terminals of the link are near ground level. The question as to the propagation conditions of millimeter waves in vegetation has recently been investigated both experimentally and theoretically. A primary goal was to obtain information on the range dependence of millimeter-wave beams in woods and forests, on beam broadening, and on depolarization. These effects are of particular interest from the viewpoint of millimeter-wave communication, i.e., point-to-point transmission into and through woods and vegetation. Typical radar problems such as target detection in vegetation clutter and backscatter from foliage at millimeter-wavelengths have been studied by Currie et al. [1], Hogan [2], Ulaby [3], and others and were not a primary subject of the present investigation.

Manuscript received October 9, 1987; revised December 30, 1987.

F. K. Schwering is with U.S. Army Cecom, Center for $\mathrm{C}^{3}$ Systems, Fort Monmouth, NJ 07703.

E. J. Violette and R. H. Espeland are with the U.S. Department of Commerce, NTIA/ITS, Boulder, CO 80303.

IEEE Log Number 8820747.
The experiments were conducted at three frequencies simultaneously, i.e., at $9.6,28.8$, and $57.6 \mathrm{GHz}$. The first of these frequencies is at $X$-band and may be regarded as a reference; the two remaining frequencies are close to the 35- and $60-\mathrm{GHz}$ bands, which are primarily of interest for millimeter-wave communication. A major problem with regard to propagation experiments in woods and forests is the physical description of this structurally very complex medium, in particular at millimeter-wave frequencies where all forest constitutents, including leaves and pine needles, have dimensions large compared to a wavelength and may have a significant effect on the propagation conditions. In addition, vegetation type and density are not uniform in a forest and may show significant variations over the transmission path. To eliminate some of this uncertainty and conduct the experiments under well-defined, reproducible conditions, a regularly planted, well groomed stand of trees of the same species and about the same growth was chosen as the test site. Because of tree size, foliage density, and humid climate, a pecan orchard near Wichita Falls, Texas, was selected as best suited for the test series. The experiments were repeated, over the same paths, in early spring and in summer, i.e., in April when the trees were without leaves and in August when they were in full leaf. Measurements were taken over path lengths from approximately 0.1 to $0.9 \mathrm{~km}$ with one to 35 trees on path and at terminal heights of 1,4 , and $6 \mathrm{~m}$ using a $\mathrm{CW}$ signal format.

In support of the experimental study, a theory of millimeter-wave propagation in woods and forests was developed. The theory describes vegetation as a random medium of discrete scatterers and characterizes wave propagation in this medium by the transport equation, i.e., by the theory of radiative energy transfer. The main purpose of the theory was to establish overall trends and assist in the interpretation of the experimental results, and a comparatively simple vegetation model was used. Important results of the theoretical study include that the range dependence of millimeter waves in vegetation is not determined by a simple exponential decrease in signal strength at constant attenuation rate; but a high attenuation rate at short distances into woods transforms into a much reduced attenuation rate at large distances. In the transition region, substantial beam broadening occurs. These results have been confirmed by the experiments and are explained by the theory in terms of the interplay of the coherent and the incoherent field component. 
In the following, a review of the field experiments conducted near Wichita Falls, Texas, is presented followed by a summary of the theory. Comprehensive reports on the experiments can be found in [4]-[6] and on the theory in [7], [8].

\section{Equipment and Test Site}

The millimeter-wave instrumentation that was used in the test series operates at three coherent frequencies: 9.6, 28.8 , and $57.6 \mathrm{GHz}$. Beamwidths of the transmit antennas were $10^{\circ}$ at all frequencies. Receive antenna beamwidths were $4.8^{\circ}$ at the $9.6-\mathrm{GHz}$ frequency and $1.2^{\circ}$ at 28.8 and $57.6 \mathrm{GHz}$. All antennas could be readily set for vertical or horizontal polarization, allowing co-polarized or cross-polarized measurements at linear polarization. A $60-\mathrm{dB}$ dynamic range limited by the last IF amplifier stage and a minimum sensitivity of $-100 \mathrm{dBm}$ was available at all frequencies. For easy mobility, the transmit and receive terminals were housed in two small vans that also carried extendable masts so that the height of the antennas could be adjusted anywhere between 1 and $6 \mathrm{~m}$ above ground. All measurements were conducted at the three test frequencies simultaneously.

The segment of the Chitwood Pecan Orchard near Wichita Falls, Texas, used for the experiments was a wellestablished and well-groomed stand of trees. The trees were planted in a square grid, with a tree spacing of approximately $13 \mathrm{~m}$, and have now attained heights of 8 to $10 \mathrm{~m}$. The maximum span of branches reached 10 to 13 $\mathrm{m}$ filling the space between rows in many areas. The initial branching occurs at a height of 1.5 to $2.0 \mathrm{~m}$. When the trees are in leaf, the weight of the new growth of twigs and nuts causes the branches to droop, such that some foliage extends down to the $1-\mathrm{m}$ level. The leaves of the pecan trees grow in clusters of 10 to 12 leaves on each twig. The leaves are arranged on the twig in balanced symmetry and range in size from $5 \mathrm{~cm}$ long to $2 \mathrm{~cm}$ wide near the branch to $20 \mathrm{~cm}$ long and $5 \mathrm{~cm}$ wide at the end of the twig. The ground was clean looking and free of weeds and underbrush.

The pictures in Fig. 1 were taken in early April when the grove was without leaves. (a) shows the uniform planting of the trees. In (b), the receiver is located with one tree on path. In (c), the scene includes the receiver van looking toward the transmitter with several trees on path. The unit on the tripod provides the 9.6-GHz phaselocked signal. An arrow points to the transmitter site. The pictures in Fig. 2 were taken in the middle of August. (a) shows the full leaf condition of the orchard and (b) shows the van behind one tree, nearly hidden by leaves. The view in (c) is taken from the side showing the van and mast. The receiver antennas are at a 6-m height. The orchard extended many hundreds of meters on the sides and back of the test area.

\section{Measurements}

For most of the experiments, the transmit terminal was located in an open field adjacent to the orchard, at a dis-

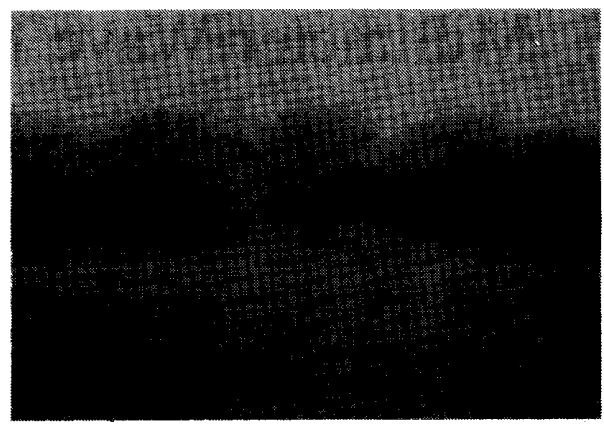

(a)

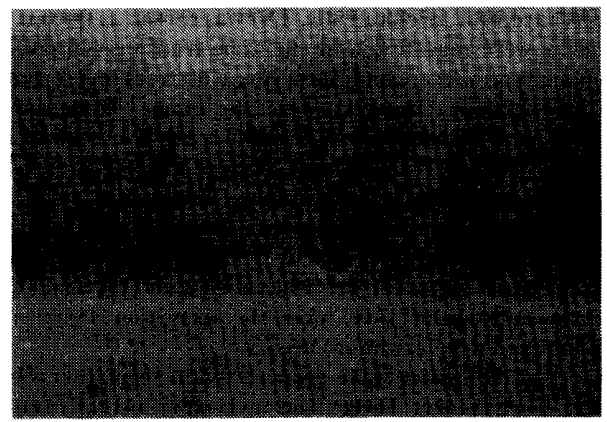

(b)

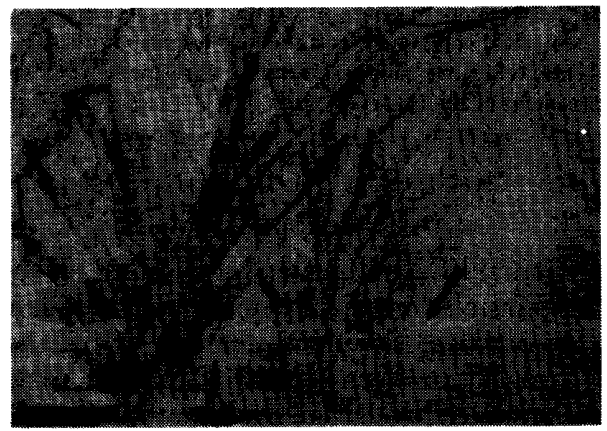

(c)

Fig. 1. Photograph of the test orchard taken in April 1982. The trees are without leaves.

tance of about $300 \mathrm{~m}$ from the first line of trees. The receive terminal was placed inside the orchard, at various positions, and usually such that the line-of-sight followed one row of trees. For each test run, the transmit and receive antennas were placed at equal heights above ground so that the line-of-sight followed a horizontal path. As mentioned earlier, three heights were used, i.e., 1, 4, and $6 \mathrm{~m}$. At the 4- and 6-m heights, significant branching was present and vegetation density was fairly high while mostly trunks were exposed at the 1-m height.

\section{A. Vegetation Loss Versus Path Length}

Measurements were made with 1, 3, 8, 11, 14, 20, 23, and 35 trees obstructing the signal path. For each receiver location and terminal height, the antennas were carefully positioned on a line that passed through the center of the tree row so that the measurements were taken over paths 


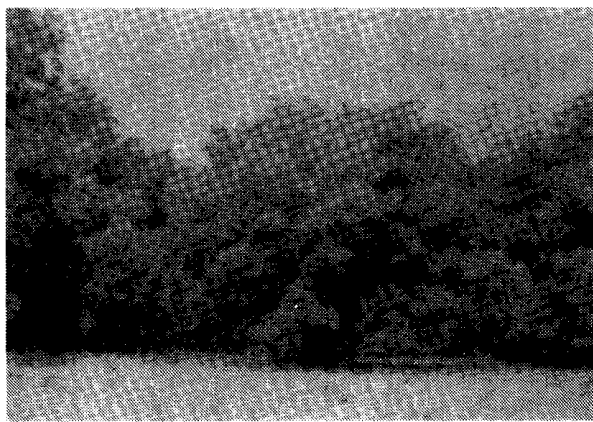

(a)

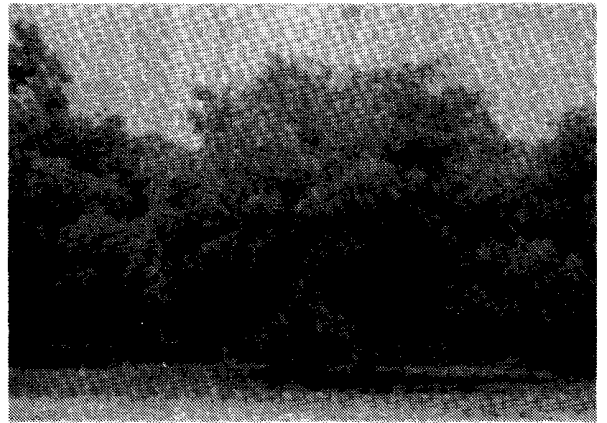

(b)

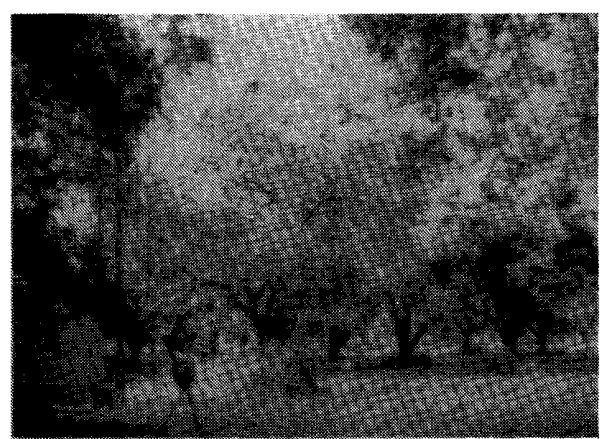

(c)

Fig. 2. Photograph of the test orchard taken in August 1982. The trees are in full leaf.

of maximum vegetation density. At each position, an azimuth scan of the receiving antennas was performed over an angular range of $\pm 15^{\circ}$ and an elevation scan over $\pm 10^{\circ}$. The received signal level was recorded. The highest level that occurred on either the azimuth or elevation scan is the value that was used in determining the path loss as a function of the number of trees on path. Fig. 3 shows results; Fig. 3(a) applies to the $1-\mathrm{m}$ height, Fig. 3(b) to the 4-m height, and Fig. 3(c) to the 6-m height. The solid curves in these figures show the vegetation loss for trees with leaves and the dashed curves for trees without leaves.' Each plotted value is normalized relative to

'The vegetation loss curves in Fig. 3 are average' curves. For each tree depth several independent measurements (up to seven) of the highest re ceived signal level were made, and the median values of these data sets were used in plotting the curves of Fig. 3. free space, i.e., the signal attenuation due to path length ( $\mathrm{d}^{-2}$-dependence) and atmospheric absorption (at 57.6 $\mathrm{GHz}$ ) has been removed and only the vegetation loss is shown. The $0-\mathrm{dB}$ level corresponds to a location of the receive terminal at the edge of the orchard.

Of particular interest is the behavior of the vegetation loss curves for the 4- and 6-m heights where the line-ofsight passed through the canopy region of the trees. In these curves an abrupt break occurs, in particular in those for trees in leaf. At small vegetation depths, with only a few trees on path, the attenuation rate is high; at large vegetation depths, it is much reduced, and the transition between the two regimes is very sharp. It is assumed that this break occurs when the dominant propagation mode changes from the strongly attenuated direct-path mode to a multiple-scatter mode that is much less attenuated; see the theoretical discussion in Section IV.

For trees without leaves, the break occurs after about eight trees; for trees with leaves, it occurs after three trees and is very pronounced. With no leaves, the small twigs and branches absorb and scatter the electromagnetic waves at a comparatively low rate so that multiple scattering does not become the dominant mode of propagation until after several trees. With leaves there is much higher absorption and scattering per unit volume so that the transition takes place with fewer trees in the path but at a much greater loss. ${ }^{2}$ The attenuation rate in the foliated state is as high as 12 to $15 \mathrm{~dB}$ per tree for the first three trees at the $4-\mathrm{m}$ height and 12 to $20 \mathrm{~dB}$ per tree at the $6-\mathrm{m}$ height. But it is only about 0.5 to $0.7 \mathrm{~dB}$ per tree at larger vegetation depths.

The curves for the 1-m height, Fig. 3(a), show that the vegetation loss for a signal path in the trunk region of the trees is significantly smaller than for a path through the canopy region, although in the trunk region the line-ofsight was obstructed by the tree trunks. The behavior of these (trunk-region) loss-curves is discussed in [5], [6]. Note that the curves for the two millimeter-wave frequencies show a higher loss after one tree than after three trees. This holds for both foliage states and is due to the peculiar propagation mechanism prevailing in the trunk region where scattering from the lower portion of the canopy may provide a significant contribution to the signal received by a narrow-beam antenna at a tree depth of three and more trees.

Figs. 3(a)-(c) show that the vegetation loss increases with frequency. This increase is noticable but not very strong in the case of trees without leaves. For trees with leaves, on the other hand, one observes in the canopy region a much higher loss at the two millimeter-wave frequencies than at $9.6 \mathrm{GHz}$. The loss at 28.8 and $57.6 \mathrm{GHz}$ seems to be nearly the same. It appears that the path loss in (summer) foliage increases significantly at the transition from the microwave to the millimeter-wave region while further increases, within the millimeter-wave re-

\footnotetext{
'The higher loss may not entirely be due to the presence of leaves. The higher moisture content of twigs and branches during summer is likely to be a contributing factor.
} 


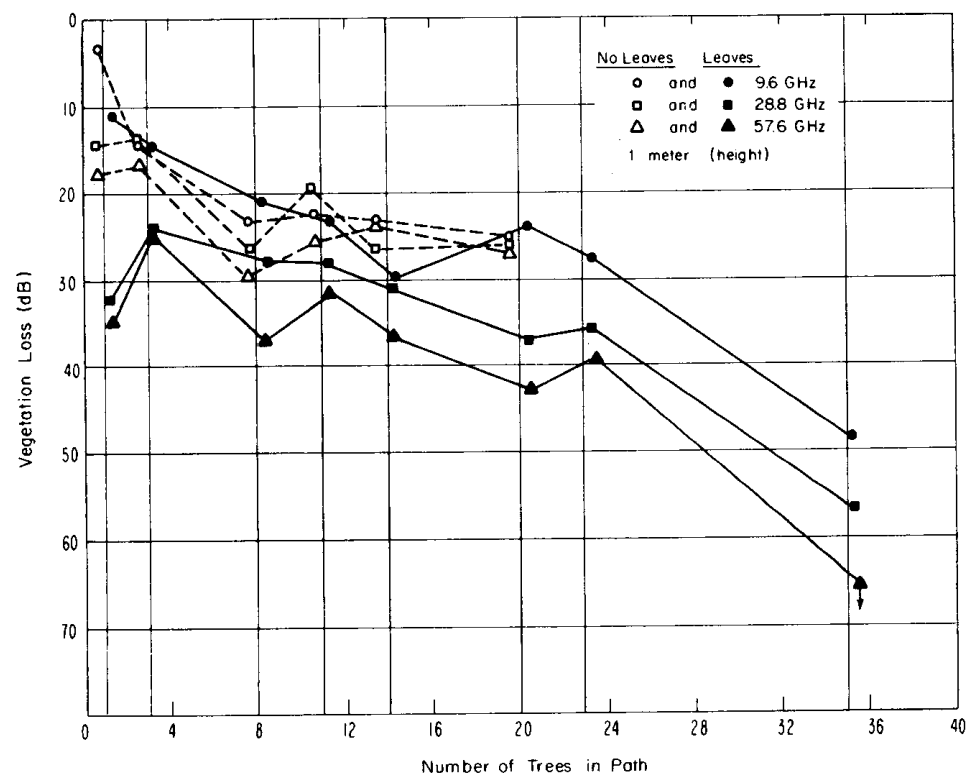

(a)

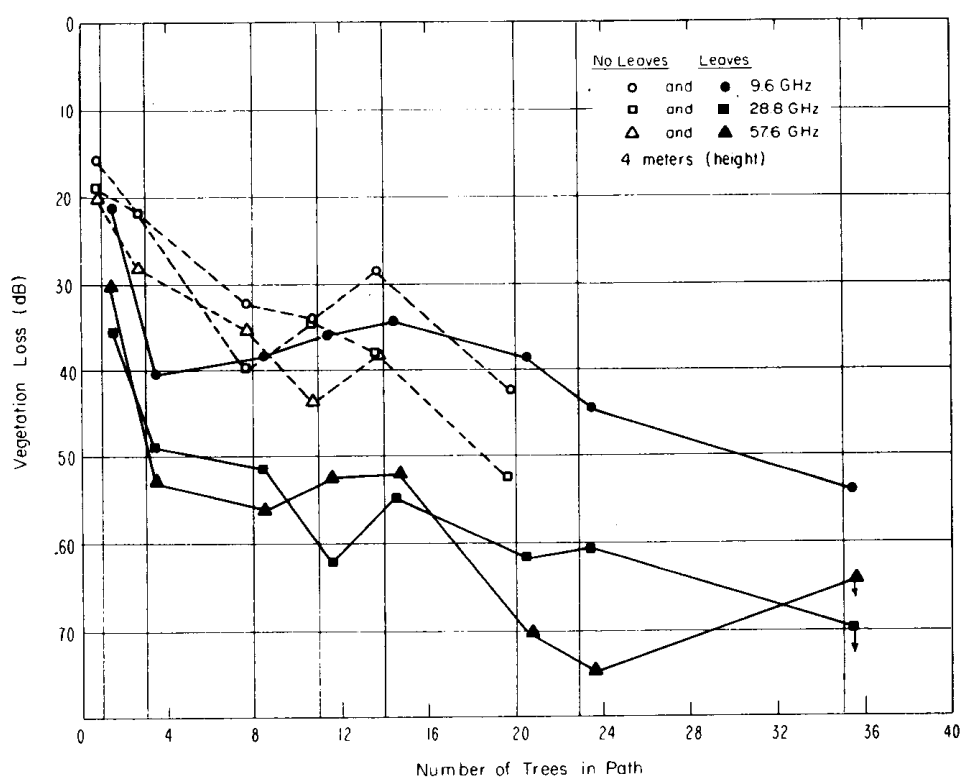

(b)

Fig. 3. Vegetation loss as a function of the number of trees on path. (a) At a height of $1 \mathrm{~m}$. (b) At a height of $4 \mathrm{~m}$. (c) At a height of $6 \mathrm{~m}$. The dashed curves apply to trees without leaves, the solid curves to trees with leaves.

gion, are slow, at least in the frequency band up to 60 $\mathrm{GHz}$. The reason for this frequency behavior is not obvious and requires further study. It is probably related to the fact that most vegetation elements, including leaves, twigs, and branches, have dimensions in the order of a wavelength in the microwave region but are large compared to a millimeter-wavelength.

At each tree depth and height, measurements were taken at vertical-to-vertical (VV) and horizontal-to-horizontal (HH) polarization. No consistent change in signal level due to polarization was detected and any polarization dependence is presumed small compared to the dependence on other variables such as transmission distance and scan angle. Since the scatter surfaces in vegetation have practically random orientations (at least when viewed in a millimeter-scale) this result was to be expected. 


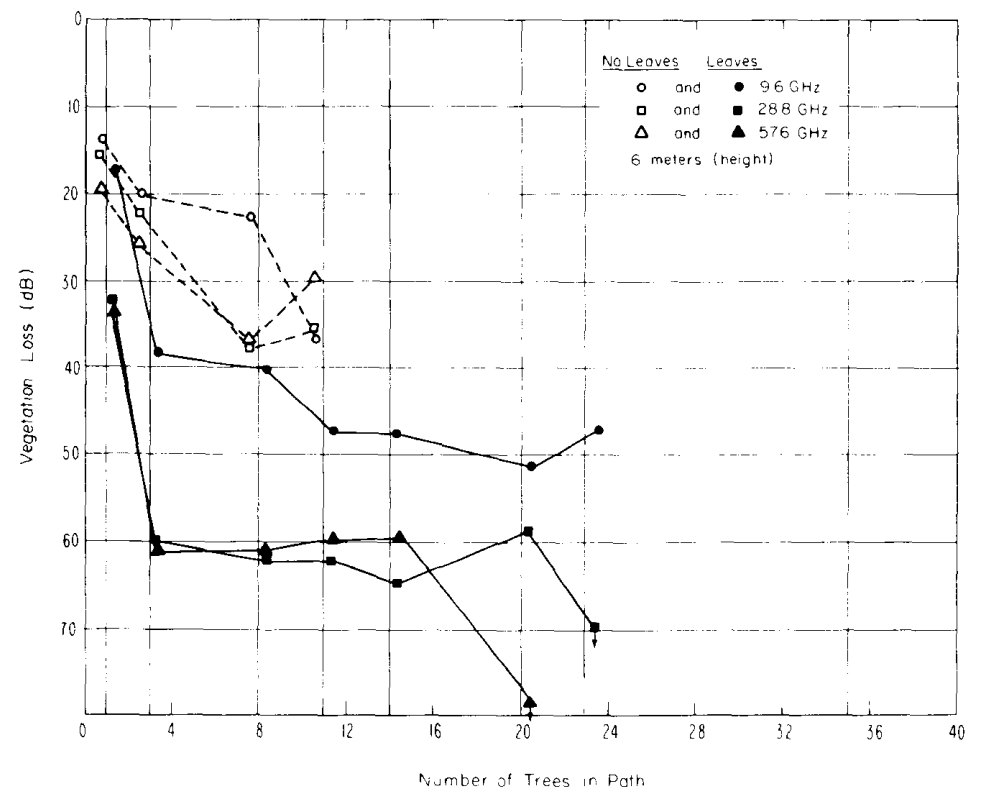

(c)

Fig. 3. (Continued)

\section{B. Azimuth Angle Scans}

As mentioned above, at each receiver location an azimuth and elevation scan of the receive antennas was performed. Fig. 4 shows typical results of azimuth angle scans at the $4-\mathrm{m}$ height. The scan range was $\pm 15^{\circ}$ with $0^{\circ}$ indicating the line-of-sight direction. The data plots show the received power versus scan angle for all three frequencies and both foliage stages at vegetation depths of $1,3,8$, and 11 trees, indicating how the directional spectrum of the signal transmitted through the orchard varies with tree depth. A reference scan, taken over an unobstructed path, is superimposed (light trace) over each data plot in the figures. The reference scan was taken at VV-polarization over a $300-\mathrm{m}$ path at a height of $1 \mathrm{~m}$ and in effect shows the radiation patterns of the receiving antennas.

Scan data recorded for trees without leaves are plotted in Fig. 4(a). The plots show a gradual loss of signal directivity (beam broadening) with increasing vegetation depth. At the 11-tree depth, the signal level is nearly flat over the $\pm 15^{\circ}$ scan range for all three frequencies. For trees in leaf, Fig. 4(b), the loss of directivity develops somewhat faster and the flattening of the signal level occurs at the 8-tree depth. A flat signal level suggests that the energy arriving at the antenna is scattered nearly equally from the entire tree. At the $4-m$ height, the receiving antenna was generally 2 to $4 \mathrm{~m}$ away from the branches but 12 to $14 \mathrm{~m}$ from the trunk of the last tree on the path. ${ }^{3}$ The $\pm 15^{\circ}$ azimuth scans cover nearly the full

${ }^{3}$ Most receiver locations were chosen at a point in the orchard where a dead tree had not been replaced, which made positioning of the receiver van much easier than trying to locate between adajacent trees. width of the tree except for some branches that may be partially obscured at the extreme outer edge.

Elevation angle scans, over an angular range of $\pm 10^{\circ}$. which were performed at each receiver location in addition to the azimuth scans have resulted in signal strengthversus-scan angle plots very similar to those obtained from the azimuth scans; the plots may be found in [5]. At a terminal height of $4 \mathrm{~m}$ and at the maximum elevation angle of $+10^{\circ}$, the receiving antenna was pointed at the highest branches of the nearest tree and, as the antenna was scanned down, its axis passed through many layers of branches before it intersected the ground at the $-10^{\circ}$ pointing. No unexpected signal peaks occurred during these scans, which is mentioned here to point out that no over-the-top mode or down-the-row mode (along a path of reduced vegetation density) was detected.

Data plots of azimuth and elevation angle scans for the 1 - and $6-\mathrm{m}$ heights may also be found in [5]. The plots for the 6-m height are very similar to those of Fig. 4, except that the signal loss is somewhat higher and the signal directivity flattens out somewhat earlier. This corresponds to the fact that at the 6-m height, the tree span is greatest and the foliage depth correspondingly increased. The azimuth scan plots for the 1-m height show, as an interesting feature, that the maximum signal typically arrives from an off-line-of-side direction, i.e., from a direction at one side or the other of the mainlobe of the reference scans. The likely reason is the complexity of the signal path that may run partially through the lower portion of the canopy of preceding trees while the line-ofsight is obstructed by a row of tree trunks that are neither perfectly aligned nor uniform in size. 

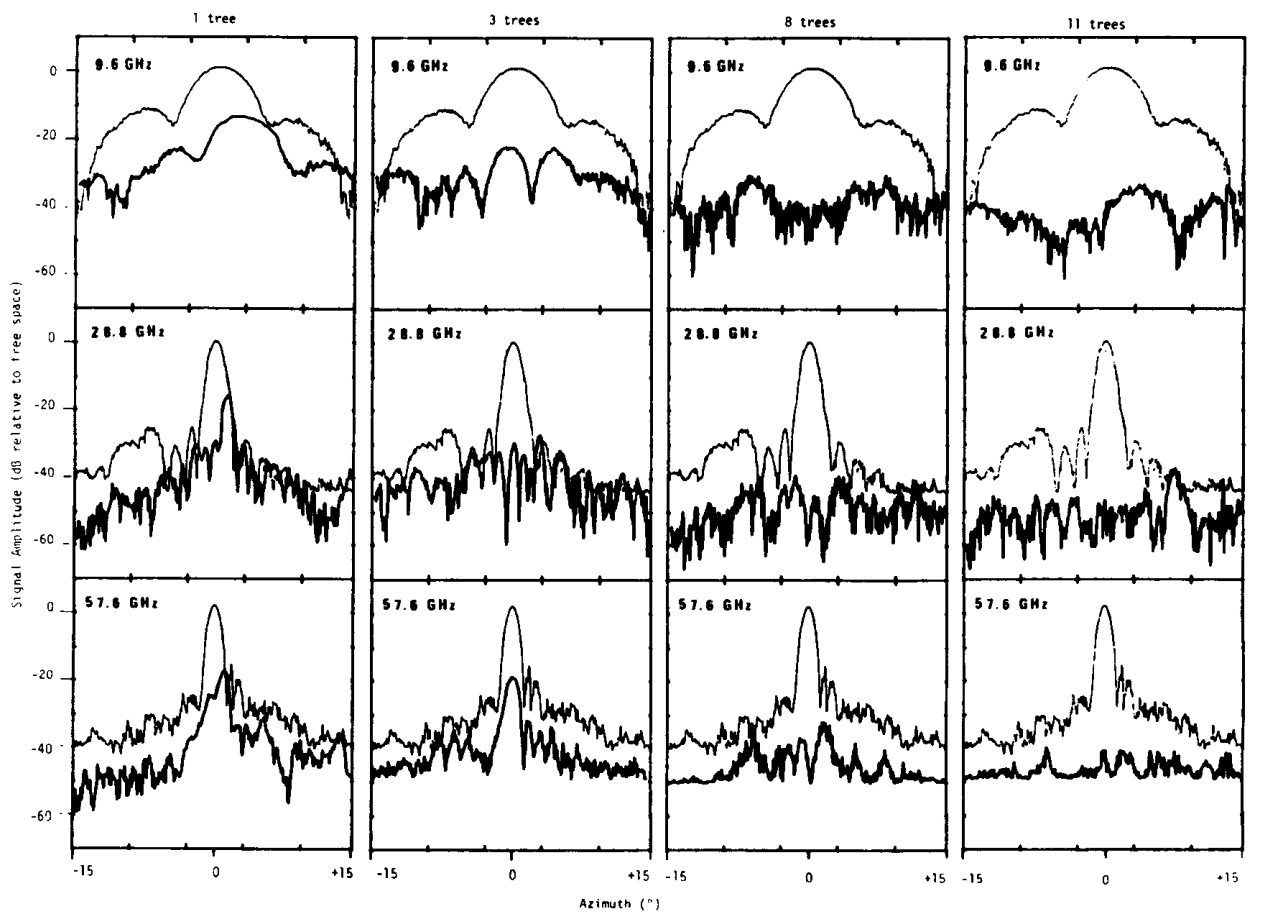

Height $=4$ meters

No lecres

(a)
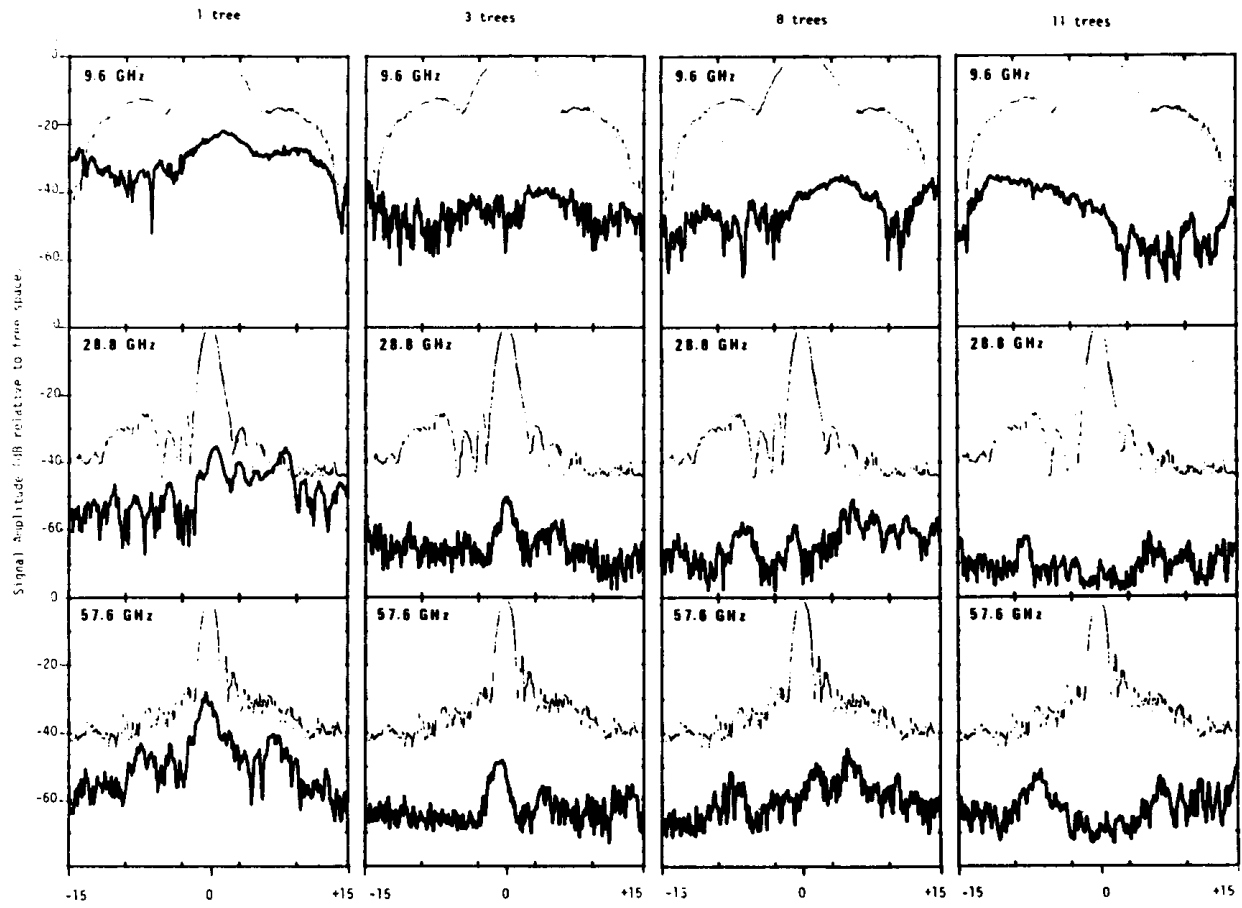

Height $=4$ meters

Leaves.

(b)

Fig. 4. Received power versus scan angle for azimuthal antenna scans at height of $4 \mathrm{~m}$. (a) Trees without leaves. (b) Trees with leaves. The received power is shown in decibels relative to free space. The light traces indicate the power received over an unobstructed path. 


\section{Horizontal Displacement Scans}

A millimeter-wave beam propagating in vegetation, in particular at large foliage depths, may be thought of as consisting of many multiscattered wavetrains propagating in various directions so that strong interference effects (multipath fadings) will occur. These effects are apparent in the data plots of Fig. 4. To further investigate these interference effects and the associated spatial fluctuations in signal strength, lateral scan measurements were made with the antennas in the orchard fastened to a track that allowed movement in the horizontal direction (at right angles to the line-of-sight). The received signal intensity was recorded while the antennas were moved from $0.7 \mathrm{~m}$ to the left of center to $0.7 \mathrm{~m}$ to the right of center. In the case of these measurements the transmitter and receiver locations were interchanged, i.e., the transmit terminal was located (and laterally scanned) in the orchard while the receive terminal was positioned about $300 \mathrm{~m}$ from the first row of trees in the open field.

Fig. 5 shows typical results obtained for a terminal height of $4 \mathrm{~m}$ and for three trees with and without leaves in the transmission path. It is obvious from the figure that considerable and fast variations in signal intensity occur as the position of the antennas in the orchard is varied, and that the correlation distance of millimeter waves in a forest environment is very short. Note that the antennas have aperture dimensions of several wavelengths so that an averaged intensity is received whose fluctuations with antenna position are already smoothed out to a certain degree. $^{4}$

The same test set-up with the receiving antennas stationary outside the orchard and the transmit antennas performing a horizontal scan inside the orchard was used also to obtain information on the depolarization of millimeterwave beams in vegetation. The receive antennas were operating at vertical polarization and the transmit antennas performed two test runs in each case, one at vertical polarization and the second at horizontal polarization. Fig. 6 shows results measured at a height of $4 \mathrm{~m}$ for a transmission path running through eight trees in full leaf. Evidently the cross-polarized component (HV) is strong at this vegetation depth. For the portions of the scans where the HV and VV signal levels are nearly equal, one can assume that the received signal was fully depolarized in good approximation.

\section{THEORY}

The main purpose of the theoretical study was to understand the basic propagation effects associated with millimeter-wave transmission through vegetation, and to assist in the interpretation of the measured results. Accordingly, a relatively simple model was developed that includes a number of approximations but can be treated analytically to a large extent. Considering the structural complexity of the vegetation medium, development of an

${ }^{4}$ This may be somewhat easier to see by interchanging (in thought) the transmit and receive functions of the antennas and by invoking reciprocity.

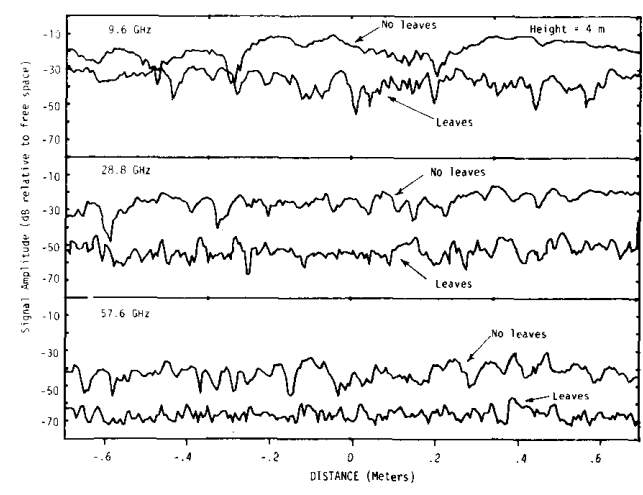

Fig. 5. Received power versus lateral displacement for horizontal antenna scan at height of $4 \mathrm{~m}$ with three trees on path. The received power is shown in decibels relative to free space. The transmit terminal was in the orchard and the transmit antennas were scanned; the receive terminal was outside the orchard.

accurate theory would be extremely difficult and require large scale computer evaluations. A theory of this type was not attempted here.

\section{A. Formulation of the Problem}

In the theoretical study vegetation is treated as a random medium of discrete, lossy scatters, and wave propagation in this medium is described in terms of the theory of radiative energy transfer (transport theory). This theory allows us to take multiple scattering fully into account and, therefore, is well suited for the present purpose. Interference effects, on the other hand, are suppressed, which is justified according to the experimental results as long as one is not interested in very local effects. In transport theory the random medium is characterized by the absorption cross section per unit volume $\sigma_{A}$, the scatter cross section per unit volume $\sigma_{S}$, and the (power) scatter function $p\left(\hat{s}, \hat{s}^{\prime}\right)$ where the unit vectors $\hat{s}$ and $\hat{s}^{\prime}$ indicate, for each scatter event, the scatter direction and the incidence direction, respectively. The scatter function is often referred to as "phase function" and the relative magnitude of $\sigma_{A}$ and $\sigma_{S}$ is described by the "albedo" $W=$ $\sigma_{S} /\left(\sigma_{A}+\sigma_{S}\right)$.

With regard to the phase function of the vegetation medium, we make the following assumptions. First, observing that the scattering surfaces in vegetation have practically random orientations, it is assumed that the scatter function is isotropic in the sense that its value will depend only on the angle $\gamma=\cos ^{-1}\left(\hat{s} \cdot \hat{s}^{\prime}\right)$ subtended by the incidence direction and the scatter direction while otherwise it is independent of these directions. Secondly, since all scatter objects in vegetation including leaves and pine needles have dimensions large compared to a millimeter wavelength, the vegetation medium will produce strong forward scattering, but also a certain amount of scattering in all other directions. Accordingly, the scatter function is assumed to consist of a strong narrow forward lobe and an isotropic background. For analytical convenience, the forward lobe is assumed to have a Gaussian profile, and 


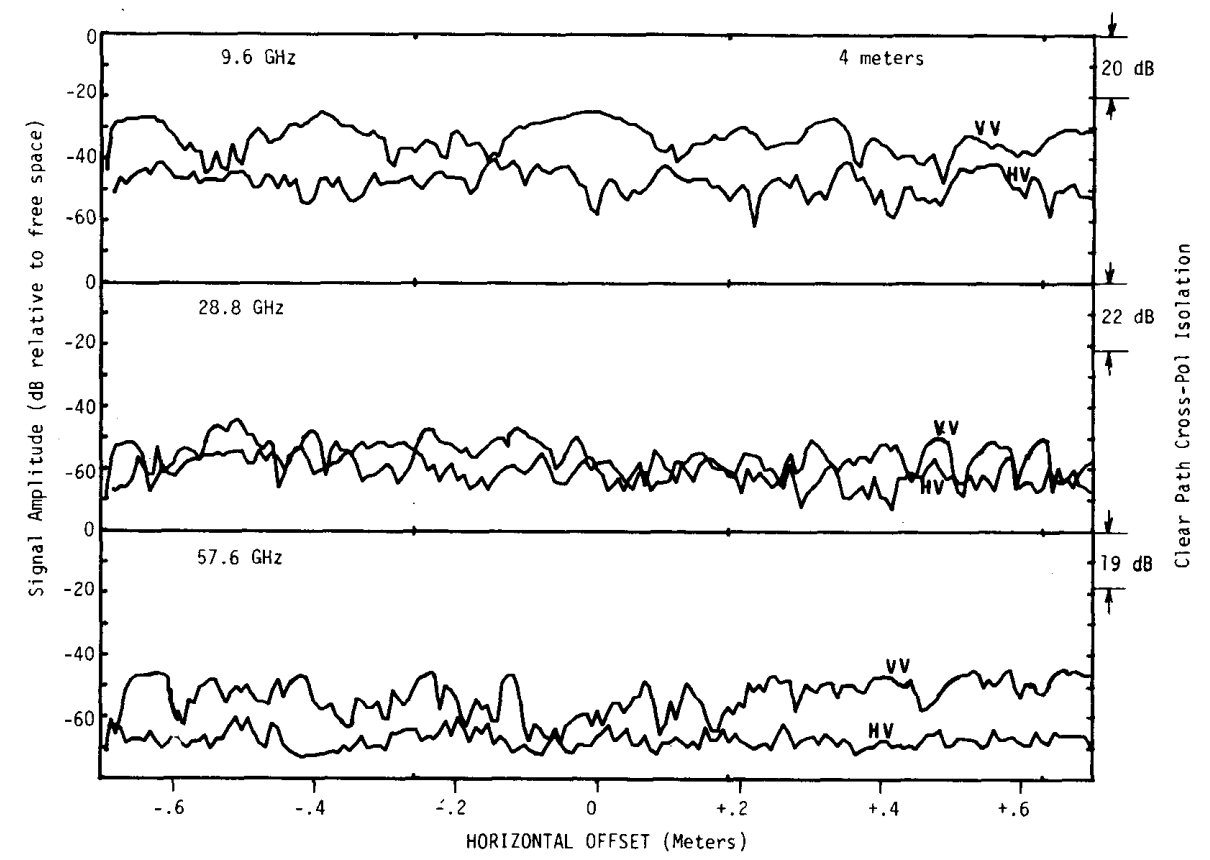

Fig. 6. Received power versus lateral displacement for horizontal antenna scan at VV-and HV-polarization. The measurements were performed at a height of $4 \mathrm{~m}$ with eight trees in full leaf on path; other conditions as in Fig. 5.

the phase function $p$ takes the form

$$
\begin{aligned}
p(\gamma) & =\alpha\left(\frac{2}{\Delta \gamma}\right)^{2} e^{-(\gamma / \Delta \gamma)^{2}}+(1-\alpha) \\
\gamma & =\cos ^{-1}\left(\hat{s} \cdot \hat{s}^{\prime}\right)
\end{aligned}
$$

where $\Delta \gamma$ is the beamwidth of the forward lobe and $\alpha$ is the ratio of the forward scattered power to the total scattered power. The first term on the right side of (1) is the forward lobe of the phase function and the second term is the isotropic background; $p(\gamma)$ is normalized such that

$$
\iint_{4 \pi} p(\gamma) d \Omega=4 \pi
$$

where $d \Omega$ is the differential solid angle about the scatter direction $\hat{s}$.

Thus, we characterize the vegetation medium by four (global) parameters: $\sigma_{A}, \sigma_{S}, \Delta \gamma$, and $\alpha$. In actuality, of course, the forest medium consists of scatter objects with a wide variety of sizes and shapes and practically random orientations, and its scatter characteristic will be very complicated, requiring many parameters for its description. The characterization of the medium by the four global parameters $\sigma_{A}, \sigma_{S}, \Delta \gamma$, and $\alpha$ assumes that a certain averaging process is permissible in the determination of the overall propagation conditions in this medium. This is assumed here without proof.

The problem that is analyzed is shown in Fig. 7. A plane wave is incident from an air half space upon the planar

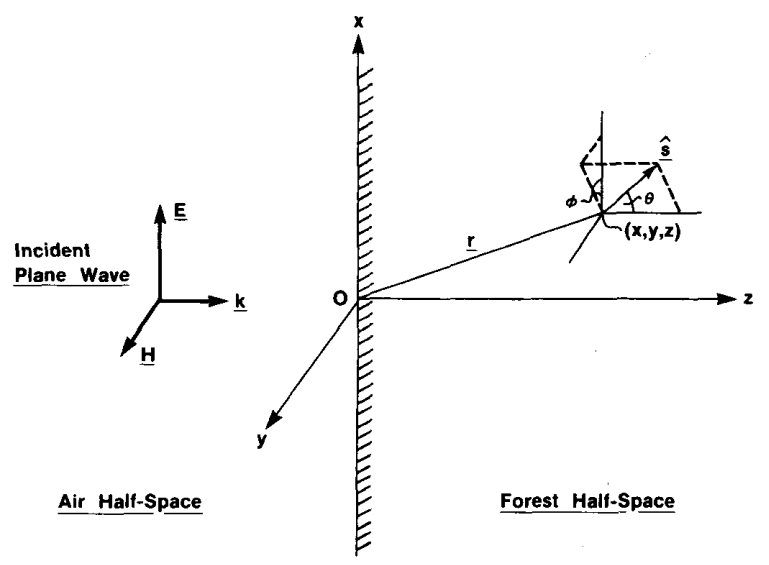

Fig. 7. Forest halfspace illuminated by plane wave.

interface of a forest half space; normal incidence is assumed. The forest medium is assumed to be statistically homogeneous, i.e., the parameters $\sigma_{A}, \sigma_{S}, \Delta \gamma$, and $\alpha$ are regarded as constants that do not vary throughout the medium. In the experimental study this situation was approximated in an overall sense by choosing a regularly planted stand of trees of the same species as the test site. Since in the experiments the transmit antennas were placed at a large distance from the edge of the orchard, the incident phase fronts were nearly planar and the assumption of an incident plane wave in the theory appears reasonable. 
The transport theory formulation of the problem takes the form

$$
\begin{aligned}
& \cos \theta \frac{\partial I}{\partial z}(z, \theta)+\left(\sigma_{A}+\sigma_{S}\right) I(z, \theta) \\
& =\frac{\sigma_{S}}{4 \pi} \int_{0}^{2 \pi} \int_{0}^{\pi} p(\gamma) I\left(z, \theta^{\prime}\right) \sin \theta^{\prime} d \theta^{\prime} d \phi^{\prime}, \\
& \quad \text { for } z>0
\end{aligned}
$$

where $I(z, \theta)$ is the specific intensity of the field in the forest medium. Because of the symmetry of the problem, $I$ varies with $z$ and $\theta$ only. The coordinates $x, y, z$, and the local directional coordinates $\theta, \phi$ are indicated in the figure. The phase function $p(\gamma)$ is given by (1) and the scatter angle follows from the relation

$$
\begin{aligned}
\cos \gamma & =\hat{s} \cdot \hat{s}^{\prime} \\
& =\cos \left(\phi-\phi^{\prime}\right) \sin \theta \sin \theta^{\prime}+\cos \theta \cos \theta^{\prime}
\end{aligned}
$$

where $\theta^{\prime}, \phi^{\prime}$ is the incidence direction for each scatter event.

There are two boundary conditions. In the interface plan $z=0$, the specific intensity is that of the incident wave (for all forward directions), i.e.

$$
I(0, \theta)=\frac{S_{p}}{2 \pi} \frac{\delta(\theta)}{\sin \theta}, \quad \text { for } 0 \leq \theta \leq \frac{\pi}{2}
$$

where $S_{p}$ is the Poynting vector (magnitude) of the incident plane wave and the symbol $\delta$ denotes the Dirac delta function. At large distances into the forest the specific intensity approaches zero since the medium is lossy. Hence

$$
I(z, \theta) \rightarrow 0, \quad \text { for } z \rightarrow \infty .
$$

With these boundary conditions (2) can be solved uniquely.

\section{B. Solution}

The solution method is explained in [7], [8]. Here we restrict ourselves to showing the results. Mathematical formulas are given first, followed by their interpretation in physical terms and the presentation of some numerical data.

In the usual manner [9], the specific intensity $I$ is split into the reduced incident intensity $I_{r i}$ and the diffuse intensity $I_{d}$, where $I_{r i}$ can be interpreted as the coherent field component and $I_{d}$ as the incoherent field component. With the particular phase function assumed in this paper, it is convenient, furthermore, to split $I_{d}$ into two parts, $I_{1}$ and $I_{2}$, where $I_{1}$ is determined primarily by scattering into the forward lobe of the phase function and $I_{2}$ by scattering into the isotropic background. Thus

$$
I=I_{r i}+I_{d}=I_{r i}+I_{1}+I_{2} \text {. }
$$

In [7], [8] it is shown that

$$
I_{r i}(z, \theta)=\frac{S_{p}}{2 \pi} \frac{\delta(\theta)}{\sin \theta} e^{-\tau}, \quad \text { for } z>0
$$

$$
I_{1}(z, \theta)=\frac{S_{p}}{4 \pi} e^{-\tau} \sum_{m=1}^{M} \frac{1}{m !}(\alpha W \tau)^{m} q_{m}(\theta), \quad \text { for } z>0
$$

$$
\text { where } \tau=\left(\sigma_{A}+\sigma_{S}\right) z
$$

$$
\begin{array}{r}
q_{m}(\theta)=\frac{1}{m}\left(\frac{2}{\Delta \gamma}\right)^{2} \exp \left[-\frac{1}{m}\left(\frac{\theta}{\Delta \gamma}\right)^{2}\right] \\
I_{2}(z, \theta)=\frac{S_{p}}{2 \pi} A \frac{s-1}{s-\cos \theta} e^{-\tau^{\prime}}, \quad \text { for } z \rightarrow \infty \\
\text { where } \tau^{\prime}=\frac{\sigma_{A}+(1-\alpha) \sigma_{S}}{s} z .
\end{array}
$$

The expression for $I_{r i}$ is exact, the expression for $I_{1}$ is an approximation which should be of good accuracy as long as the forward lobe of the phase function is narrow $(\Delta \gamma$ $<\pi$ ), and the expression for $I_{2}$ is an asymptotic representation that holds for large distances into the medium where $I_{2}$ is the dominant field component. At short distances, $I_{2}$ has to be determined by numerical methods.

In (6a), $M$ is a sufficiently large integer, and in (7a), $A$ $=A\left(W^{\prime}\right)$ and $s=s\left(W^{\prime}\right)$ are an amplitude factor and an eigenvalue, respectively, that have to be computed numerically. Table I shows values for $s$; note that $s$ is always greater than unity. The reduced albedo $W^{\prime}$, which determines $A$ and $s$, differs from the conventional albedo $W=$ $\sigma_{S} /\left(\sigma_{A}+\sigma_{S}\right)$ in that the scatter cross section $\sigma_{S}$ is reduced by multiplication with $1-\alpha$. (In any scatter event, only the fraction $1-\alpha$ of the scattered power is transferred into the omnidirectional background and contributes to $I_{2}$, while the remainder is scattered into the forward direction). Thus

$$
W^{\prime}=\frac{(1-\alpha) \sigma_{S}}{\sigma_{A}+(1-\alpha) \sigma_{S}}=\frac{(1-\alpha) W}{1-\alpha W} .
$$

The coherent field component (5) dominates at short distances into the forest halfspace. It has a well defined direction of propagation (that of the incident plane wave) and may be viewed as the continuation of the incident plane wave into the random medium, where it decreases exponentially due to absorption and scatter. Its attenuation rate $\tau / z=\sigma_{A}+\sigma_{S}$ is equal to the extinction cross section of the forest medium.

The incoherent component $I_{d}=I_{1}+I_{2}$ is generated by the scattering of the coherent component and may be thought of as consisting of many single-and multi-scattered wavetrains propagating in various directions; it is characterized by a directional spectrum rather than a well defined direction of propagation. Its range dependence is determined by a (linear) increase in magnitude at short distances into the forest half space and by an exponential decrease at large distances. This holds for both $I_{1}$ and $I_{2}$.

The field component $l_{1}$ is the dominant component at intermediate distances into the vegetation medium. Equation (6a) represents $I_{1}$ as a series showing the various or- 
TABLE I

Eigenvalue $S$ Versus Reduced Albedo $W^{\prime}$

\begin{tabular}{cccccccccccc}
\hline \hline$W^{*}$ & 0.0 & 0.25 & 0.50 & 0.60 & 0.70 & 0.80 & 0.85 & 0.90 & 0.95 & 0.99 & 1.00 \\
\hline 5 & 1.0 & 1.00067 & 1.0444 & 1.102 & 1.207 & 1.408 & 1.589 & 1.903 & 2.635 & 5.797 & $\infty$
\end{tabular}

ders of multiple scattering. Note that the functions $q_{m}(\theta)$, which determine the "beam shape" of the series terms, have the same general form as the forward lobe of the phase function but their beamwidth, $\sqrt{m} \Delta \gamma$, broadens out with $m$. The dominant term at any given distance $z$ into the medium is the term of order $m \simeq \alpha W \tau$ and the beamwidth of $I_{1}$ increases roughly with $\sqrt{z}$. In [7], [8] it is shown, furthermore, that the attenuation rate of $I_{1}$ decreases with $z$, from $\sigma_{A}+\sigma_{S}$ at small vegetation depths to $\sigma_{A}+(1-\alpha) \sigma_{S}$ at large depths. In particular, in a strongly forward scattering medium where $\alpha$ is close to unity and $\sigma_{S} \gg \sigma_{A}$, the change of attenuation rate will be substantial. Comparison of the theory with the experimental data (see Section IV-C) indicates that the vegetation medium at millimeter-wave frequencies has these characteristics.

The field component $I_{2}$ has the smallest amplitude and the lowest attenuation rate and will be the dominant component at large distances into the forest halfspace. In a strongly scattering medium with $\sigma_{S} \gg \sigma_{A}$ the reduced albedo $W^{\prime}$ can be close to unity and the eigenvalue $s$ can exceed unity by a considerable margin; see (7c) and Table I. Thus, the attenuation rate of $I_{2},(7 \mathrm{~b})$, will be significantly smaller than that of $I_{r i}$ and appreciably smaller than that of $I_{1}$. Equation (7a) shows, moreover, that the beamwidth of $I_{2}$ is wide, in particular if $s$ is large.

In summary, at short distances into the forest half space the coherent field component dominates. This field component has a narrow beamwidth and a high attenuation rate. It decreases due to both absorption and scattering. The incoherent component, on the other hand, decreases, in effect, due to absorption only, while scattering reproduces the incoherent component. ${ }^{5}$ Its attenuation rate is determined by absorption only, but by absorption over multiscattered ray paths of extended length (as determined by $W$ and $\alpha$ ). It can be shown that the attenuation rate of the incoherent component is always larger than $\sigma_{A}$ but, as discussed above, it is smaller (and often significantly smaller) than $\sigma_{A}+\sigma_{S}$, the attenuation rate of the coherent component. Hence, at a certain distance into the forest halfspace, the coherent (direct path) component will disappear within the incoherent component which will take over leading to a multiscatter propagation mode of substantially reduced attenuation rate and increased beamwidth.

A similar range dependence in woods and forests, i.e., a transition from a high attenuation rate at short distances

\footnotetext{
${ }^{5}$ This applies, in particular, in the case considered here that the inciden field is a plane wave that illuminates the entire boundary plane of the forest half space so that side scattering out of the beam cannot occur. For an incident beam of finite cross section such side scattering is likely to in crease the attenuation rate of the incoherent component.
}

to a much lower rate at large distances has also been observed at much lower frequencies, i.e., for propagation of VHF signals through dense vegetation (jungles) [10]. The underlying mechanism, however, is completely different. The change of attenuation rate of VHF signals is attributed to the so-called up-over-down propagation mechanism where a lateral wave is excited which travels along the horizontal interface between the forest canopy and the air region above it (Tamir theory). Since this wave propagates mostly in the air region, it is much less attenuated than the direct-wave traveling through the forest and will dominate at larger distances.

Note that the effect is determined by the coherent field component alone while the incoherent component is negligible at wavelengths in the VHF region. Conversely, a lateral wave supported by the canopy-air interface should not be expected at millimeter-wavelengths. The interface is extremely rough in a millimeter scale; and no indication of such wave has been observed in the experiments (see Section III-B).

\section{Numerical Results}

Figures 8 and 9 show numerical results. The situation is that of Fig. 7 where it is assumed that a narrow-beam receiving antenna is placed into the forest halfspace; the quantity $Q$ is the received power in decibels normalized to the maximum power that is received when the antenna is placed into the boundary plane $z=0$ and aligned with the incoming radiation. The antenna is assumed to have a Gaussian radiation pattern with a half-power beamwidth equal to that of the millimeter-wave antennas used in the experiments $\left(1.2^{\circ}\right)$. The half-power beamwidth of the forward lobe of the phase function of the medium is chosen to be $6^{\circ}$ so that it exceeds the beamwidth of the antenna by a factor of 5 . (The size of the test antennas was significantly larger than the cross section of most scatter objects in a forest environment with the exception, perhaps, of tree trunks.) The distance into the forest halfspace is given in the figures in terms of the normalized vegetation depth $\tau=\left(\sigma_{A}+\sigma_{S}\right) z$.

Figure 8 shows the range dependence of the received power, i.e., $Q=Q(\tau)$ for various values of $W$. The curves demonstrate the change in attenuation rate from a high value at small $\tau$ to a lower value at large $\tau$. The effect is pronounced, in particular, for strongly scattering media with $\sigma_{S} \gg \sigma_{A}$ where the albedo $W$ is close to unity. Increasing $\alpha$, the ratio of the forward scattered power to the total scattered power, would have a similar effect.

Evidently the theoretical range dependence curves are in good qualitative agreement with the experimental curves of Fig. 3, although the transition between the two regions of high and low attenuation rate appears to be much sharper in the measured curves for trees in leaf.

The curves $Q=Q\left(\theta_{R}\right)$ of Fig. 9 show the dependence of the received power on the pointing direction $\theta_{R}$ of the receiving antenna. It is assumed here that the antenna is placed at various vegetation depths $\tau$ where it performs an angular scan over the range $0 \leq \theta_{R} \leq 20^{\circ}$. 


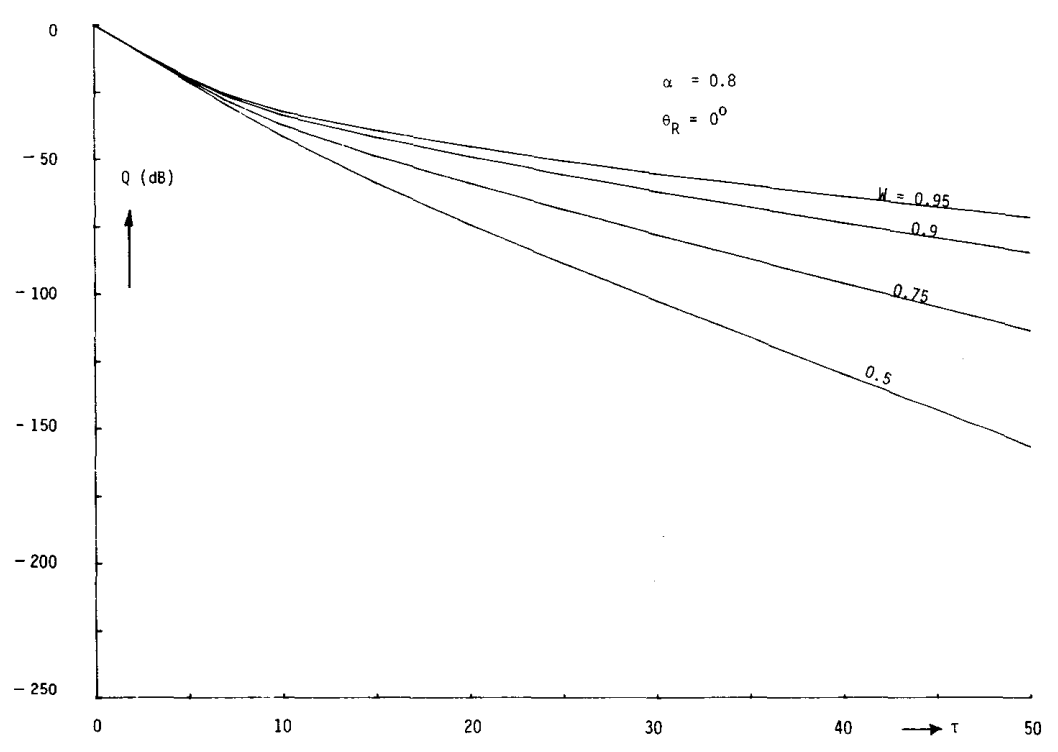

Fig. 8. Range dependence of received power (theory).

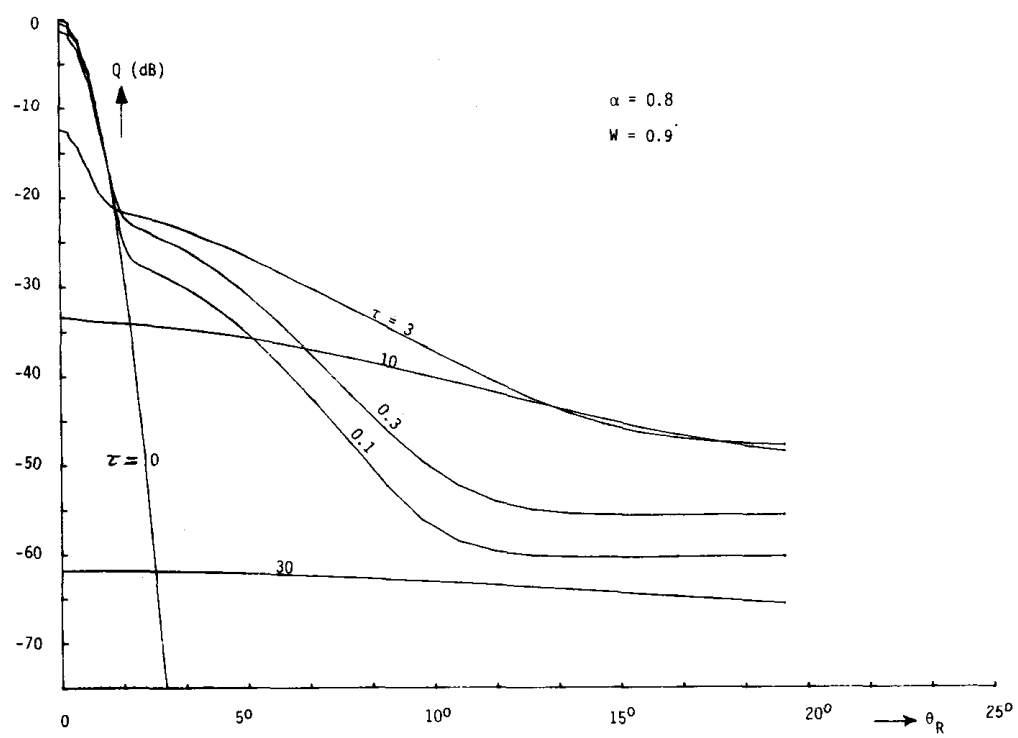

Fig. 9. Directional dependence of received power (theory).

For $\tau=0$, when the receiving antenna is positioned in the interface plane, the received field is an unperturbed plane wave, and $Q\left(\theta_{R}\right)$ traces the Gaussian radiation pattern of the receiving antenna. As $\tau$ is increased, beam broadening occurs. For small $\tau=0.1$ and 0.3 , the coherent component remains dominant, as indicated by the narrow maximum about $\theta_{R}=0$. But the incoherent component produces a broad tail consisting of a peak of moderate height and width extending to about $\theta_{R}=10^{\circ}$, and a very broad background. The peak is determined by $I_{1}$, and the background by $I_{2}$. For $\tau=3$, the coherent component is still clearly visible, but for $\tau=10$ it has all but disappeared within the incoherent component. At $\tau=30$, the incoherent component has taken over completely, the signal level has dropped significantly, and the beam has broadened out substantially. The curves of Fig. 9 apply to $\alpha=0.8$ and $W=0.9$ but in their general behavior are typical also for other $\alpha$ and $W$ values.

Again, good qualitative agreement of theoretical and experimental results is observed. The measured curves of Fig. 4 indicate that for short distances into the orchard, i.e., at vegetation depths of one and three trees, the coherent component is significantly present as evidenced by the narrow maximum of the scan pattern. At larger veg- 
etation depths, corresponding to propagation paths through eight and eleven trees, the coherent component has disappeared within the incoherent component that dominates the received power, resulting in a reduced signal amplitude and substantial beam broadening.

The scintillations in the measured curves of Fig. 4 are interference effects (fadings); the exact locations of the maxima and minima should vary rapidly with antenna position so that, in an averaged curve, these fluctuations will disappear. Since transport theory suppresses interference effects, the corresponding theoretical curves of Fig. 9 are smooth.

The four parameters $\sigma_{A}, \sigma_{S}, \Delta \gamma$, and $\alpha$ by which the theory characterizes the medium are determined by the microscopic and macroscopic structure of the forest and, in principle, can be derived from a detailed physical description of the medium. But, because of the considerable complexity of the forest environment, this would be a very difficult task. ${ }^{6}$

A more promising approach would be the following. Given the good qualitative agreement between theory and experiments one may now try to obtain quantitative agreement by choosing the numerical values of $\sigma_{A}, \sigma_{S}, \Delta \gamma$, and $\alpha$ such that a best fit of the calculated curves to the measured curves is achieved. At the same time, this procedure would yield order of magnitude estimates for the four parameters. A curve fitting method of this type and the conclusions one would draw from it, of course, have to be taken with a great deal of caution since the theoretical model contains strong simplifications and is not identical with the experimental situation.

An attempt to fit the theoretical range dependence curves to the measured curves of Fig. 3 is discussed in [7], [8]. The curves measured at heights of 4 and $6 \mathrm{~m}$ above ground were used for this comparison. Reasonably good agreement may be obtained for trees without leaves, and, at short and large distances into woods, for trees with leaves. But the rather sharp transition between the high and low attenuation regimes, experimentally observed for trees in leaf, is difficult to model with the theory, at least in its present form. One trend seemed to be consistent, however; in order to obtain reasonable agreement, the parameters $\alpha$ and $W$ had to be chosen close to unity, i.e., in the $0.9-0.95$ region, which would indicate that forward scattering is indeed a significant effect in millimeter-wave propagation in vegetation and that scattering dominates over absorption, with $\sigma_{S}$ being, by a factor of 10 to 20 ,

${ }^{6}$ Models for electromagnetic wave propagation in vegetation based on a detailed analysis of the scatter and absorption properties of forest elements such as leaves, branches, and tree trunks have been developed by Brown [11] and by Lang et al. [12]. But these models apply to the UHF and microwave regions where the cross-sectional dimensions of most forest elements are in the order of or smaller than a wavelength, and only the forward scattered incoherent field component has to be considered (in addition to the coherent field component). In the millimeter-wave region, all forest elements, including leaves and pine needles, have dimensions large compared to a wavelength. One is dealing here with a very different situation and, as pointed out above, the incoherent, multiscattered field componen is important and must be fully taken into account. larger than $\sigma_{A}$. Also this observation, of course, is subject to further examination.

\section{CONCLUSIONS}

\section{A. Experiments}

Propagation measurements in vegetation were conducted with CW signals at $9.6,28.8$, and $57.6 \mathrm{GHz}$ to obtain information on signal attenuation, beam broadening, and depolarization as a function of vegetation depth. A well established, uniformly planted pecan tree orchard was chosen as the test site because it provided well defined, reproducible test conditions and a foliage density that was very constant with distance. For a propagation path through the trunk region of the orchard $(1-\mathrm{m}$ height with no underbrush) and through the canopy region (4and 6-m heights ) with trees in a defoliated state, the signal strength decreases exponentially with distance into the orchard at a nearly constant attenuation rate, i.e., the vegetation loss curve (loss in decibels) decreases in a nearly linear fashion with the number of trees on path. In contrast, for a propagation path through the canopy region with the trees fully in leaf, the vegetation loss curve shows an abrupt break at a foliage depth of about $30 \mathrm{~m}$ that corresponds to three trees on path. For the first $30 \mathrm{~m}$ through foliage, the increase in vegetation loss with foliage depth is nearly linear at a rate of $1.3-2.0 \mathrm{~dB} / \mathrm{m}$, depending on frequency, while at foliage depths beyond $30 \mathrm{~m}$ the loss increases at a rate that averages only $0.05 \mathrm{~dB} / \mathrm{m}$. Azimuth and elevation angle scans of the receiving antenna have shown, furthermore, that at vegetation depths just past the break in the vegetation loss curve substantial beam broadening occurs.

The theory interprets these effects as resulting from the interplay of the direct path propagation mode (coherent field component), which dominates at short distances into vegetation but is strongly attenuated, and a multiple scattering propagation mode (incoherent field component) that has a much reduced attenuation rate and a much increased beamwidth, and that dominates at large distances into vegetation.

The measured data also shows a clear trend for the vegetation loss to increase with frequency. This increase seems to occur in a smooth but not necessarily uniform fashion. In particular, for trees in leaf, the foliage loss increases substantially as the frequency is raised from 9.6 to $28.8 \mathrm{GHz}$. But it increases at a much slower rate between 28.8 and $57.6 \mathrm{GHz}$, the additional loss amounting to a small correction only.

Measurements at vertical-to-vertical and horizontal-tohorizontal polarizations did not show any consistent difference in received signal levels; the propagation conditions in vegetation seem to be polarization independent in the $10-$ to $60-\mathrm{GHz}$ band. But, as expected, substantial depolarization occurs at large vegetation depths.

\section{B. Theory}

A theory of millimeter-wave propagation in woods and forests was derived that models vegetation as a random 
medium of discrete, lossy scatters and characterizes wave propagation in this medium by the theory of radiative energy transfer. Theoretical results are in good qualitative agreement with measured data; both show the same trends. The theory includes a number of simplifications, however, and a conclusive, quantitative correlation of theoretical and measured data will require a refinement of the theory. Also, a larger experimental data base would be helpful for this purpose.

\section{REFERENCES}

[1] N. C. Currie, E. E. Martin, and F. B. Dyer, "Radar foliage penetration measurements at millimeter wavelengths," Engineering Experiment Station, Georgia Institute of Technology, Atlanta, GA, Final Rep. EES/GIT Project A-1485-100, Dec. 1975.

[2] D. L. Hogan, "An analysis of foliage effects on long-range surveillance," MIT Lincoln Laboratory, Lexington, MA, Tech. Note 19809, Apr. 1980.

[3] F. T. Ulaby, "Vegetation clutter model," IEEE Trans. Antennas Prop., vol. AP-28, pp. 538-545, July 1980

[4] E. J. Violette, R. H. Espeland, A. R. Mitz, F. A. Goodknight, and F. Schwering, "SHF-EHF propagation through vegetation on Colorado east slope," U.S. Army CECOM, Fort Monmouth, NJ, R\&D Tech. Rep. CECOM-81-CS020-F, June 1981.

[5] E. J. Violette, R. H. Espeland, and F. Schwering, "Vegetation loss measurements at $9.6,28.8$, and $57.6 \mathrm{GHz}$ through a pecan orchard in Texas," U.S. Amy CECOM, Fort Monmouth, NJ, R\&D Tech. Rep. CECOM-83-2, Mar. 1983

[6] - "Vegetation loss measurements at $9.6,28.8$ and $57.6 \mathrm{GHz}$ through a pecan orchard in Texas,"' in Proc. Int. Symp. Multiple Scattering Waves Random Media Random Rough Surfaces, V. V. Varadan and V. K. Varadan, Eds., The Pennsylvania State University, July 19-Aug. 2, 1985.

[7] R. A. Johnson and F. Schwering, "A transport theory of millimeter wave propagation in woods and forests," U.S. Army CECOM, Fort Monmouth, NJ, R\&D Tech. Rep. CECOM-TR-85-1, Feb. 1985.

[8] F. Schwering and R. A. Johnson, "A transport theory of millimeter wave propagation in woods and forests," Journal of Wave-Material Interaction, vol. 1, pp. 205-235, Apr. 1986.

[9] A. Ishiman, Wave Propagation and Scattering in Random Media. New York: Academic, 1978, vol. 1, ch. 7.

[10] T. Tamir, "On radio-wave propagation in forest environments," IEEE Trans. Antennas Prop., vol. AP-15, pp. 806-817, Nov. 1967.

[11] G. S. Brown and W. J. Currie, "A theory and model for wave propagation through foliage," Radio Sci., vol. 17, July-Aug. 1982.

[12] R. H. Lang, A. Schneider, F. J. Altan, and Kiet Truong, "UHF radio wave propagation through forests,"'Fort Monmouth, NJ, R\&D Tech. Rep. CECOM-81-0136-6, May 1984

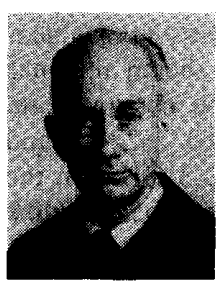

Felix Schwering (M'60-SM'86) was born on June 4,1930 , in Cologne, Germany. He received the Dipl. Ing. degree in electrical engineering and the Ph.D. degree from the Technical University of Aachen, West Germany, in 1954 and 1957, respectively.

From 1956 to 1958, he was Assistant Professor at the Technical University of Aachen. In 1958 he joined the U. S. Army Research and Development Laboratory at Fort Monmouth, NJ, where he performed basic research in free space and guided propagation of electromagnetic waves. From 1961 to 1964 he worked as a Member of the Research Staff of the Telefunken Company, Ulm, West Germany, on radar propagation studies and missile electronics. In 1964 he returned to the U. S. Army Electronics Command, Fort Monmouth, NJ, and has since been active in the fields of electromagnetic-wave propagation, diffraction and scatter theory, theoretical optics, and antenna theory. Recently he has been involved, in particular, in millimeter-wave antenna and propagation studies. From 1969 to 1978, he was leader of the Antenna Research Team of the Center of Communication Systems at Forth Monmouth and since 1979 has been a member of the Research Council of this Center.

Dr. Schwering is a member of SIGMA XI and a Visiting Professor at Rutgers University and at New Jersey Institute of Technology. $\mathrm{He}$ is a member of URSI, Commission B, and recipient of the 1961 and 1982 Best Paper Awards of the IEEE Antennas and Propagation Society (jointly with G. Goubau). During the year 1984/85, he worked with the US Army Research Office, Research Triangle Park, NC, under the Visiting Laboratory Associates Program.

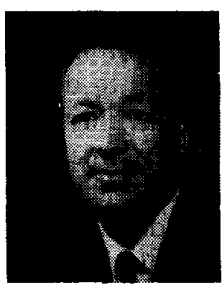

Edmond J. Violette was born in Deer Lodge, MT, on September 25, 1930. He received the B.S. degree in electrical engineering from Montana State University, Bozeman, in 1953.

From 1953 to 1956 he served in the Air Force Wright-Patterson Air Development Center, Dayton, OH. In 1956, he joined the Central Radio Propagation Laboratory where beginning in 1963 he developed the first computer-controlled digital ionospheric monitor with on-line data processing. Beginning in 1969 he was involved in the measuring and recording of the effects on the ionosphere of the Platteville Ionospheric Heating facility. In 1974 he began experimental work concerning atmospheric effects on micro/millimeter wave and optical propagation including very high data rate links $(1 \mathrm{~Gb} / \mathrm{s})$.

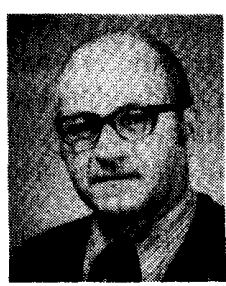

Richard $\mathbf{H}$. Espeland was born in Grenora, ND, on July 14,1928 . He received the B.S. degree in electrical engineering from Montana State University, Bozeman, in 1957 and the M.S. degree in telecommunications from the University of Colorado, Boulder, in 1976.

From 1957 to 1966 he worked in the Electronics Research Division of the U.S. Naval Ordnance Laboratory, Corona, California. He then joined the Telecommunications Laboratory of ESSA at Boulder, Colorado. Since 1977, he has been working in the EM Propagation Measurements and Analysis Group of the Institute for Telecommunication Sciences where he has been studying millimeter wave line-of-sight link performance as well as millimeter wave propagation through vegetation and in an urban environment. 